\title{
COMMON FEATURES IN THE FLOW STRUCTURE AND TURBULENCE OF TIP LEAKAGE FLOWS IN AXIAL TURBOMACHINES
}

\author{
Joseph Katz \\ William F. Ward Sr. Distinguished Professor of Engineering at the Department of Mechanical Engineering, \\ Whiting School of Engineering, at Johns Hopkins University
}

\begin{abstract}
Tip leakage flows adversely affect the overall performance of axial turbomachines, and are major contributors to noise, vibrations, onset of stall in compressors, and cavitation breakdown in pumps. Consequently, considerable efforts have already been invested in studying them, and developing techniques to alleviate these undesirable effects. Measurements of flow within rotating machinery have been a challenge due to limitations in visual access to the interior of rotor passages and reflections from boundaries. We have resolved this problem by constructing a unique facility, in which the refractive index of transparent rotor is matched with that of the fluid a concentrated aqueous solution of sodium iodide. This arrangement facilitates unobstructed flow measurement at any point within the machine using 2D, stereo, holographic and tomographic particle image velocimetry (PIV). This presentation focuses on common features in the flow structure and turbulence in the tip region of several axial turbomachines. They have been observed in a series of experiments performed within machines with different sizes, speeds, load distributions and tip-gap sizes. These observations follow the evolution of the backward leakage flow across the narrow tip gap, its rollup into a tip leakage vortex (TLV) near the suction side of the blade, and the dynamics of this vortex within the rotor passage. Several notable phenomena include: (i) in instantaneous realizations, the vicinity of the TLV center contains multiple interlacing structures that never roll up into a single vortex; (ii) the TLV migrates from the suction side of one blade to the pressure side of the neighboring blade; (iii) vortex breakup/bursting occurs in regions of adverse pressure gradients, rapidly spreading TLV fragments over substantial fraction of the tip region; (iv) Endwall casing boundary layer separation occurs when the leakage flow meets the main passage flow, feeding counter rotating vorticity into a layer that surrounds the TLV center; (v) the (anisotropic and inhomogeneous) turbulence levels are high in the shear layer connecting the TLV to the suction-side corner of the blade, near the TLV center, and in the region of endwall boundary layer separation. Specific mechanisms dominating the turbulence production will be introduced and discussed.
\end{abstract}

